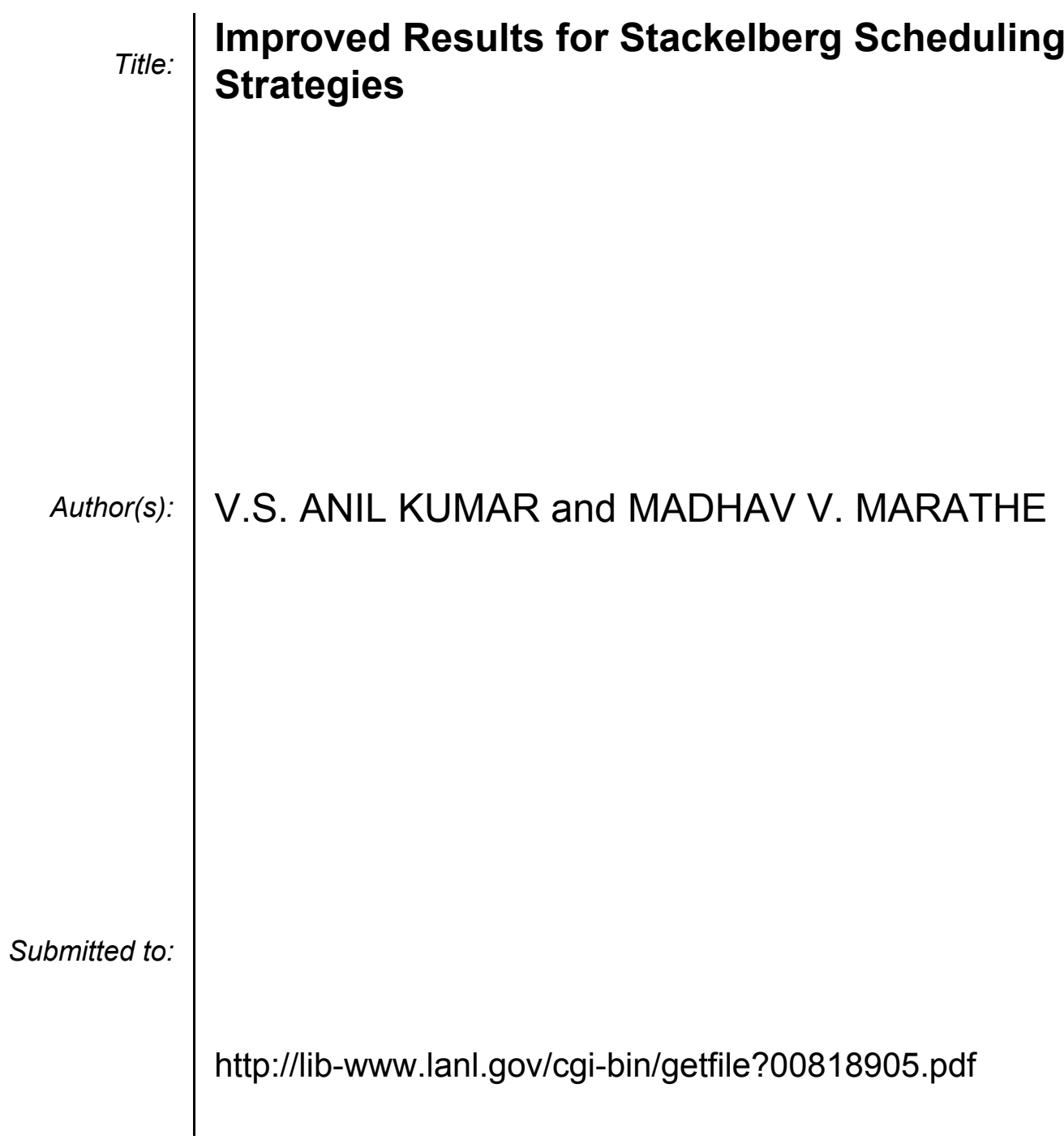
Laboratory requests that the publisher identify this article as work performed under the auspices of the U.S. Department of Energy. Los Alamos National Laboratory strongly supports academic freedom and a researcher's right to publish; as an institution, however, the Laboratory does not endorse the viewpoint of a publication or guarantee its technical correctness. 


\title{
Improved Results for Stackelberg Scheduling Strategies
}

\author{
V.S. ANIL KUMAR ${ }^{3}$ \\ MADHAV V. MARATHE ${ }^{3}$
}

November 12, 2001

\begin{abstract}
We continue the study initiated in [Ro01] on Stackelberg Scheduling Strategies. We are given a set of $n$ independent parallel machines or equivalently a set of $n$ parallel edges on which certain flow has to be sent. Each edge $e$ is endowed with a latency function $l_{e}(\cdot)$. The setting is that of a non-cooperative game: players choose edges so as minimize their individual latencies. Additionally, there is a single player who control as fraction $\alpha$ of the total flow. The goal is to find a strategy for the leader (i.e. an assignment of flow to indivual links) such that the selfish users react so as to minimize the total latency of the system. Building on the recent results in [Ro01, RT00], we show the following:

1. We devise a fully polynomial approximate scheme for the problem of finding the cheapest Stackelberg Strategy: given a performance requirement $(1+\epsilon)$, our algorithm runs in time polynomial in $n$ and $\epsilon$ and produces a Stackelberg strategy $s$, whose associated cost is within a $1+\epsilon$ factor of the optimum stackelberg strategy $s^{*}$.

The result is extended to obtain a polynomial-approximation scheme when instances are restricted to layered directed graphs in which each layer has a bounded number of vertices.

2. We then consider a two round Stackelberg strategy (denoted 2SS). In this strategy, the game consists of three rounds: a move by the leader followed by the moves of all the followers folowed again by a move by the leader who possibly reassigns some of the flows. We show that 2SS always dominates the one round scheme, and for some classes of latency functions, is guaranteed to be closer to the global social optimum. We also consider the variant where the leader plays after the selfish users have routed themselves, and observe that this dominates the one-round scheme.
\end{abstract}

Extensions of the results to the special case when all the latency functions are linear are also presented. Our results extend the earlier results and answer an open question posed by Roughgarden [Ro01].

\footnotetext{
${ }^{1}$ Basic and Applied Simulation Science (D-2) Los Alamos National Laboratory, P. O. Box 1663, MS M997, Los Alamos NM 87545. The work is supported by the Department of Energy under Contract W-7405-ENG-36. Email: anil, marathe@lanl.gov.
} 


\section{Introduction and Motivation}

The dynamic behavior of large scale networks can often be modelled by non-cooperative games, with agents acting in a selfish manner. The fixed points of such dynamical systems often correspond to Nash equilibrium of the corresponding non-cooperative game. Although Nash equilibria are adequate from the standpoint of user optimium, these operating points are usually inefficient as measured by the way system resources are used (a.k.a. system/social optimum) [Ro01, KLO97a, KLO97b]. The inefficient use of a system can be overcome by a number of possible strategies that aim to bring the operating point of the system closer to a social or a system optimum. Examples of this include:

1. Pricing: Use pricing mechanisms that lead to strategies by players with equilibria that are more efficient [CS+93, FPS00, SMG01]

2. Algorithmic Mechanisms: Network wide rules on how commodities are stored, routed and scheduled [NR99, CS00].

3. Network Design: Designing networks in which Nash equilibria are close to global optimum [KLO97b, Ro01a].

The above approaches demand either the addition of a new component to the networking structure, such as price or apriori design decisions regaring the network topology or policies used. Here we consider an alternative approach motivated by the earlier work of [KLO97a, Ro01]. In this setting, we have two types of players: set of selfish players who wish to minimize the latency they experience and a manager whose aim is to optimize the overall system and is aware of the selfish players use (called manager/leader). This property allows the manager (leader) to predict the response of the selfish users and thus can help guide the final equilibrium point that is more closer to the system optimum (in terms of the global objective function under consideration). This is an instance of a class of games wherein there is an exogeneously defined order of players such that the first player (called the leader) declares his strategy first and enforces it on the other player (the follower). Such games are referred to as Leader Follower games or alternatively as Stackelberg games.

Our original motivation to study such games arose in the context of developing large scale simulations of socio-technical systems and Strategic force planning problems. Such simulations include: TRANSIMS: a large transportation simulation project and large scale simulations of deregulated power markets. See [Web, AP+01] for more details on these projects. Game theoretic analysis in the context of transportation systems has been carried out extensively [DS69, BMW56, Wa52]. It is likely that understanding the game semantics of these problems would lead to faster and more scalable simulations.

Here we consider a particular Stackelberg game. This game has been studied extensively in the past [KP99, KLO97a, KLO97b, MS01, Ro01]. We have a single source destination pair joined by $m$ parallel links from the source to the sink. Latency functions are specified for the links, and they are required to be non-decreasing. This can also be viewed as a machine scheduling problem. Each agent is assumed to constitute an infinitesimal fraction of the flow, and the total flow to be set up is denoted by $r$. In addition, there is one distinguished player called the leader (or manager). The leader controls $\alpha$ fraction of the flow $r$. The protocol of the game is as follows: First, the leader chooses an assignment $\mathbf{s}=\left(a, \ldots, s_{m}\right)$ of flows on the links, taking into account that remaining players are going to play selfishly. Next, all the selfish players

route their flows so that the system reaches a Nash equilibrium, $t$. The assignment chosen by the leader is 
called a stackelberg strategy and it satisfies $\sum_{i} s_{i}=\alpha r$. The goal is to minimize the cost of the flow $s+t$. In this paper, that the time it takes to reach the unique Nash equilibrium is not of interest. But, we will be interested in the computational cost of finding a Stackelberg strategy. We will say more about this later.

As argued in [KP99, KLO97a, KLO97b, MS01, Ro01], despite its simplicity, the above setting models a number of practical situations that arise in the design of communication networks and machine scheduling. For example, as noted in [KLO97a], in broadband networks, bandwidth is separated among different virtual paths resulting effectively, in a system of parallel and non-interferring links. Moreover, recent IP specifications provides the option of choosing a particular paths to route their packets [CR+93, DH95]. Similarly, as noted in [BPS99], many ISPs have chosen to increase their network capacity by placing a set of parallel fiber optic links between consecutive switching centers. In this setting, the ISPs as owners of the infrastructure can reserve certain amount of bandwidth for itself and allow the remainder of the bandwidth to be used by the customers. See [KP99, KLO97a, MS01, Ro01] for other examples of such a setting.

\section{Our Contributions and Related Work}

We continue the study initiated in [Ro01] on finding polynomial time computable Stackelberg strategies that improve upon the cost of Nash equilibria obtained without the presence of any leader. In our setting, we have a total of $r$ units of flow to route and the leader controls $\alpha \cdot r$ units of flow. The main results of this paper include the following:

1. Given a set of $n$ parallel links with latency functions represented as polynomials with non-negative coefficients, we devise a family of algorithms that for a given $\epsilon>0$, output a Stackelberg Strategy $\mathbf{1 S S}_{\epsilon}$ with the following property: The cost of the solution induced by $\mathbf{1 S S}$ is no more than $(1+\epsilon)$ times the cost of the solution induced by an Optimal Stackelberg strategy. The algorithms run in time that is polynomial in the size of the network and the $\epsilon$ and thus constitute a fully polynomial time approximation scheme for computing Optimal Stackelberg Strategies. Note that as shown in [Ro01], the problem of computing the optimal Stackelberg strategies is Weakly NP-hard even for instances consisting of $n$ paralle links between a given source destination pair, even when restricted to linear latency functions on each edge. Roughgarden [Ro01] as such is interested in the quality of Stackelberg induced solution as compared to a system optimal strategy. Nevertheless, his results imply a $\frac{1}{\alpha}$ approximation algorithm for the case when no restriction ${ }^{2}$ is placed on the latency functions and a $\frac{4}{3+\alpha}$ approximation algorithm for the case of linear latency functions. The author in [Ro01] left open the question of designing approximation algorithm with a better performance guarantee

Can we do better with more sophisticated algorithms? Indeed the results do not rule out the possibility of a fully polynomial-time approximation scheme for the problem.

Thus our results answer the above question affirmatively.

2. We then cosider slightly more complicated topologies. Extending the first set of results, we devise polynomial time approximation schemes for computing computing Optimal Stackelberg Strategies, when instances are restricted to be layered directed graph of bounded width (i.e. bounded number of nodes per layer). The result infact hold when we have a constant number of multiple source destination

\footnotetext{
${ }^{2}$ Throughout this paper and the earlier work of [Ro01, RT00], it is assumed that all latency functions are non-negative, continuous and non-decreasing.
} 
pairs and the Stackelberg leader has control over a fraction $\alpha$ of the flow requirement for each pair. Moreover, the only requirement on latency functions is that they are polynomials with positive coefficients. The result also holds when we are allowed polynomially many parallel edges between any pair of nodes. Thus the result can be viewed as a strict generalization of the first result. However in contrast to the first result, the algorithm is only a PTAS as opposed to FPAS.

3. We then consider two variants of the basic Stackelberg Strategy. the first variant can be viewed as a repeated Stackelberg strategy. A natural, well known, generalization of the stackelberg strategy is to allow the manager to change his assignment. Thus the game has three basic rounds: In round 1, the leader assigns certain flow $s$ to each of the links. In round 2, the selfish players then assign the remaining $(1-\alpha) r$ flow (denoted by $t$ ) such that the flow $(s+t)$ is a Nash equilibrium. Finally, in round 3, the leader is allowed to reroute some of the $\alpha r$ flow it controls. Call this assignment $\xi$. Thus the resulting assignment is $s+t$. We call this the 2-round Stackelberg Strategy. It is straightforward to define a $k$-round Stackelberg in a similar fashion. The first observation is that more than 2 rounds do not help any more. Second, we show that 2 round Stackelberg Strategy strictly dominates the 1 round Stackelberg Strategy, i.e. the cost of assignment is no more than the cost of 1 round Stackelberg. For some special classes of latency functions, we obtain better facors. An interesting aspect of the problem is that in the instances where one and two round stackelberg strategies guaranty only a $\frac{1}{\alpha}$, even the Nash equilibrium is within $\frac{1}{\alpha}$ of the optimum.

4. Finally, we consider the case when the remaining agents first choose their assignment (denoted $t$ ) that yields a Nash equilibrium for the $(1-\alpha) r$ units of flow and then the manager chooses $s$. We show that this actually is better than one round Stackelberg $1 S S$, though in general the asymptotic factor is still $\frac{1}{\alpha}$. The result points out the relative importance of two different factors. On one hand, when the leader plays first, it imposes its strategy over the followers.

On the other hand, the leader can wait to see what the selfish users play and then try and route the remaining flow so as to minimize the total latency.

\section{Basic Model and Preliminaries}

For sake of consistency, to the extent possible, we use the notation used in [Ro01]. In general, we have a directed network $G(V, E)$, with latency functions $\ell_{e}()$ specified on each edge $e$. A vector $\bar{r}$ specifies the flow requirement between different pairs of nodes in $G$. For a function $f$, we use $f(x)$ to denote the derivative of $f$ at $x$. Here we will be concerned with latency functions $\ell_{e}()$ that are continuous, differentiable and non-decreasing ${ }^{3}$.

By $\mathbf{x}=O P T(G, r)$ and $\mathbf{y}=\operatorname{Nash}(G, r)$, we denote the optimum flow assignment and the Nash flow assignment, respectively, when the flow requirements are specified by $r$. Let as defined above $\mathbf{x}=\left(x_{q}, \ldots, x_{n}\right)$ be the (system or social) optimal assignment (i.e., a feasible assignment that minimizes $\sum_{i} x_{i} \ell_{i}\left(x_{i}\right)$ ). Order the links so that $\ell_{i}\left(x_{i}\right) \leq \ell_{j}\left(x_{j}\right), \forall i<j$. Gievn a flow assignment $\mathbf{u}$ to the links the cost associated with $\mathbf{u}$ is measured as $C(\mathbf{u})=\sum_{i} u_{i} \ell_{i}\left(u_{i}\right)$. Sometimes, we will need to consider a subset of links rather than all the links. To do this, let $X \subseteq E$ denote a subset of links. Then given an assignment $\mathbf{u}$ of flows to $E$ we use

- $\mathbf{u}_{X}$ to denote the projection of $\mathbf{u}$ on $X$,

\footnotetext{
${ }^{3}$ The conditions assumed are identical to those in [RT00].
} 
- $C\left(\mathbf{u}_{X}\right)=\sum_{i \in X} u_{i} \ell_{i}\left(u_{i}\right)$ to denote the cost of the assignment restricted to $X$ and

- $\mathbf{u}(X)=\sum_{i \in X} u_{i}$ to denote the sum of flows on links restricted to $X$.

In general, we will use $\mathbf{u}$ and $\mathbf{u}_{E}$ interchangeably.

For the most part, this paper deals with networks consisting of two nodes, $v_{s}$ and $v_{t}$, with $m$ parallel links, $1, \ldots, m$, between them. Thus the graph $G(V, M)$ consists of $V=\left\{v_{s}, v_{t}\right\}$ and edge set $M=\left\{e_{1}, \ldots, e_{m}\right\}$ with each $e_{i}=\left(v_{s}, v_{t}\right)$. In this setting, $r$ units of flow have to be sent from $v_{s}$ to $v_{t}$. Throughout this paper we will use $\mathbf{z}$ to denote a vector of flow values assigned to edges and use $z$ to denote the flow on edge $i$.

Definition 1 A Stackelberg Strategy is an assignment vector $\mathbf{s}$ such that $\sum_{i} s_{i}=\alpha r$ and the Nash equilibrium $\boldsymbol{t}^{4}$ induced by $\mathrm{s}$ is a vector satisfying the following properties.

1. $\sum_{i} t_{i}=(1-\alpha) r$

2. $\ell_{i}\left(s_{i}+t_{i}\right) \leq \ell_{j}\left(s_{j}+t_{j}\right)$ for all $i, j$ such that $t_{i}>0$.

From the definition above, given the Stackelberg assignment $\mathbf{s}$, the induced Nash assignment $\mathbf{t}$ is well defined, and the cost induced by $\mathbf{s}$ is defined as $C(\mathbf{s}+\mathbf{t})$ or $C(\mathbf{s})$ and is given by $C(\mathbf{s})=\sum_{i}\left(s_{i}+t_{i}\right) \ell_{i}\left(s_{i}+t_{i}\right)$.

An instance of the Stackelberg Routing problem is given by $(G, \alpha, r)$. Here $G$ is the graph consisting of parallel links, $\alpha$ is the fraction of the flow can be chosen by the leader and $r$ is the total flow to be routed. Thus $(1-\alpha) r$ units of flow are routed by selfish players and each controls an insignificantly small quantity of the this flow. The game is played in two steps:

1. In Step 1, the Stackelberg player (leader) chooses a flow vector $\mathbf{s}$ such that $\sum_{i} s_{i}=\alpha r$.

2. In Step 2, the selfish users route the remainder of flow i.e. choose an assignment $\mathbf{t}$ of $(1-\alpha) r$ units of flow to the links to reach a Nash equilibrium induced by $\mathbf{s}$.

The cost of the game is $C(\mathbf{s})=\sum_{i}\left(s_{i}+t_{i}\right) \ell_{i}\left(s_{i}+t_{i}\right)$. Let $\mathbf{s}^{*}$ be the optimal Stackelberg strategy, and $\mathbf{t}^{*}$ the (unique) Nash equilibrium induced by $\mathbf{s}^{*}$. Thus

$$
\mathbf{s}^{*}=\arg \min \{C(\mathbf{s}): \mathbf{s} \text { is a Stackelberg Strategy }\}
$$

As shown [Ro01], finding an $\mathbf{s}$ with the minimum associated cost is NP-complete. Thus it is natural to look for a strategy $\mathbf{s}$ having cost as close as possible to the optimal strategy. We define the notion of an approximation algorithm for such problems.

Definition 2 An $\rho$-approximation algorithm for the Stackelberg Routing problem is a polynomial time algorithm that outputs a Stackelberg strategy $\boldsymbol{s}$ such that its induced cost $C(\boldsymbol{s})$ is no more than a multiplicative factor $\rho$ more than the cost of the assignment induced by the optimal Stackelberg Strategy s, i.e. $C(\boldsymbol{s}) \leq \rho C\left(\boldsymbol{s}^{*}\right)$. A polynomial time approximation scheme for the Stackelberg Routing problem is a family of algorithms that on a given performance requirement $\epsilon$, run in time polynomial in $\epsilon$ and problem specification and output an assignment vector $\boldsymbol{s}_{\epsilon}$ such that $C\left(\boldsymbol{s}_{\epsilon}\right) \leq(1+\epsilon) C\left(\boldsymbol{s}^{*}\right)$.

Finally, we recall the results in [Ro01, RT00] that will be used in the rest of the paper.

\footnotetext{
${ }^{4}$ Technically $t$ should be indexed by $\mathbf{s}$; but in the current setting this will be clear from context and will thus be omitted.
} 
Lemma 1 ([Ro01, RT00]) Suppose $M$ is a set of machines (parallel links) with continuous, nondecreasing latency functions. Then:

1. For any rate $r>0$ of job traffic, there exists an assignment of jobs to $M$ at Nash equilibrium

2. If $\boldsymbol{x}$ and $\boldsymbol{x}$ ' are assignments at Nash equilibrium for $(M, r)$, then $\forall i \in M, l_{i}\left(x_{i}\right)=l_{i}\left(x_{i}^{\prime}\right)$.

Lemma 2 ([Ro01, RTO0]) Suppose $M$ is a set of machines (parallel links) with differentiable latency function $l$. Furthermore assume that $x_{i} l_{i}\left(x_{i}\right)$ is a convex function for each machine $i$. Then an assignment $\boldsymbol{x}$ to the machines $M$ is optimal iff $\forall i, j \in M$, if $x_{i}>0$, then

$$
l_{i}\left(x_{i}\right)+x_{i} l_{i}^{\prime}\left(x_{i}\right) \leq l_{i}\left(x_{j}\right)+x_{j} l_{j}^{\prime}\left(x_{i}\right) .
$$

Moreover, the optimal assignment can be computed in polynomial time.

In other words, all machines with positive flow assignment have the same marginal cost function.

\section{A Fully Polynomial Approximate Scheme for Stackelberg Strategies}

\subsection{Properties of $\mathrm{s}^{*}$}

We first isolate certain invariants of the optimal strategy, and show that the knowledge of these invariants reduces the problem of finding $s^{*}$ to solving a mulidimensional knapsack instance. To get a $(1+\epsilon)$-approximate solution, it is sufficient to guess these invariants, and this is demonstrated in the next section.

Let $M_{=0}=\left\{i: t_{i}^{*}=0\right\}$ and $M_{>0}=\left\{i: t_{i}^{*}>0\right\}$. The cost induced by $s^{*}+t^{*}$ is the sum of the cost of assignments on $M_{=0}$ and on $M_{>0}$. Then:

- Since $t^{*}$ is a Nash equilibrium, by Lemma 1, the latency on all $i \in M_{>_{0}}$ is the same. Let us denote this latency by $L^{*}$.

- Second, since $s^{*}$ is an optimal Stackelberg strategy, by lemma 2, the marginal costs of increasing cost on any $i \in M_{=0}$ are the same. We will denote this by $D^{*}$.

- Finally, since $\forall i \in M_{=0}, \quad t_{i}^{*}=0$ it must follow that $\forall i \in M_{=0}, \quad \ell_{i}\left(s_{i}^{*}\right) \geq L^{*}$ (otherwise, the Nash assignment would choose to add some flow on link $i$ ).

The following observation shows that the assignment of $s^{*}$ to $M_{>0}$ is not unique.

Observation 1 Let $\hat{s}$ be any assignment such that $\hat{s}_{i}=s_{i}^{*}, \forall i \in M_{=0}$ and $\hat{s}_{i} \leq s_{i}^{*}+t_{i}^{*}, \forall i \in M_{>0}$, while satisfying $\sum_{i} \hat{s}_{i}=\sum_{i} s_{i}^{*}$. Let $\hat{t}_{i}=s_{i}^{*}+t_{i}^{*}-\hat{s}_{i}, \forall i$. Then, $\hat{t}$ is a Nash equilibrium induced by the stackelberg strategy $\hat{s}$ and $\hat{s}+\hat{t}$ has the same cost as $s^{*}+t^{*}$.

\subsection{Reduction to Multidimensional Knapsack}

Assume now that we know $L^{*}$ and $D^{*}$, and $S_{0}^{*}=s^{*}\left(M_{=0}\right)$. Then $U_{>0}^{*}=r-S_{0}^{*}$ is the total assignment on $M_{>0}$ by $s^{*}+t^{*}$. Also assume that we can solve for the roots of the latency functions exactly. All these assumptions will be relaxed within a $1+\epsilon$ factor when we look for an approximate solution in the next section. 
For each link $i$, the basic difficulty is deciding whether it must belong to $M_{0}$ or to $M_{>0}$. Once this decision is made, the assignment on it is easily fixed: if $i \in M_{>0}$, solve for $u_{i}$ in $\ell_{i}\left(u_{i}\right)=L^{*}$; if $i \in M_{=0}$, solve for $s_{i}$ in $\left(s_{i} \ell_{i}\left(s_{i}\right)\right)^{\prime}=D^{*}$, where the prime denotes the derivative. The assumptions that the latency functions are polynomial and non decreasing imply that the roots are unique.

For each link $i$, we associate the tuple $\left(s_{i}^{*}, u_{i}^{*}\right)$ where $\ell_{i}\left(u_{i}^{*}\right)=L^{*}$ and $s_{i}^{*}$ is defined as follows: let $y$ be the solution to $\left(x \ell_{i}(x)\right)^{\prime}=D^{*}$. If $\ell_{i}(y) \geq L^{*}$, define $s_{i}^{*}=y$, otherwise $s_{i}^{*}=\infty$. Let $U^{*}=\sum_{i} u_{i}^{*}$.

Lemma 3 Let $X$ be a subset of links that minimises $\sum_{i \in X} s_{i}^{*} \ell_{i}\left(s_{i}^{*}\right)$, while satisfying $\sum_{i \in X} s_{i}^{*}=S_{0}^{*}$ and $\sum_{i \in X} u_{i}^{*}=U^{*}-U_{>0}^{*}$. Consider the stackelberg strategy $s$ defined as $s_{i}^{\prime}=s_{i}^{*}, \forall i \in X$ and $s_{i}^{\prime}=0, \forall i \notin X$. Then $C\left(s^{\prime}\right)=C\left(s^{*}\right)$.

Proof: Consider the assignment $t^{\prime}$ defined as: $t_{i}^{\prime}=0, \forall i \in X$ and $t_{i}^{\prime}=u_{i}^{*}, \forall i \in \bar{X}$. We first show that $t^{\prime}$ is the Nash assignment induced by $s$. By construction, $\ell_{i}\left(s_{i}^{\prime}\right) \geq L^{*}, \forall i \in X$ and $\ell_{i}\left(s_{i}^{\prime}+t_{i}^{\prime}\right)=L^{*}, \forall i \in \bar{X}$. This implies that $\ell_{i}\left(s_{i}^{\prime}+t_{i}^{\prime}\right)=L^{*}$ for all $i$ such that $t_{i}^{\prime}>0$. By choice of $X, s^{\prime}(X)=S_{0}^{*} \leq \alpha r$ and $t^{\prime}(\bar{X})=U_{>0}^{*}=r-S_{0}^{*}$. Thus, $t^{\prime}$ satisfies all the constraints of the Nash equilibrium induced by $s^{\prime}$.

Recall that $C\left(s^{\prime}\right)=C\left(s^{\prime}+t^{\prime}\right)=\sum_{i \in X} s_{i}^{\prime} \ell_{i}\left(s_{i}^{\prime}\right)+\sum_{i \notin X} t_{i}^{\prime} \ell_{i}\left(t_{i}^{\prime}\right)$. Since $\ell_{i}\left(t_{i}^{\prime}\right)=L^{*}, \forall i \notin X$, we have $\sum_{i \notin X} t_{i}^{\prime} \ell_{i}\left(t_{i}^{\prime}\right)=U_{>0}^{*} L^{*}=C_{M_{>0}}\left(s^{*}\right)$. Since $X$ is a feasible set minimizing $\sum_{i \in X} s_{i}^{*} \ell_{i}\left(s_{i}^{*}\right)$, it follows that $C_{X}\left(s^{\prime}\right) \leq C_{M_{=0}}\left(s^{*}\right)$. Together, these two observations imply $C\left(s^{\prime}\right)=C\left(s^{*}\right)$.

Note that the stackelberg assignment does not need to assign anything on $\bar{X}$. The problem of finding such an $X$ is a variation of the standard knapsack problem, and can be solved in pseudopolynomial time by dynamic programming, which is sketched here briefly. The next section will modify it to obtain a polynomial time approximation.

As mentioned before, each link $i, i=1, \ldots, m$, is associated with a pair $\left(s_{i}^{*}, u_{i}^{*}\right)$ and $\operatorname{cost} c_{i}=s_{i}^{*} \ell_{i}\left(s_{i}^{*}\right)$, and we are given $S_{0}^{*}, U_{0}^{*}$. We need to compute the cheapest subset, $X$, satisfying $s^{*}(X)=S_{0}^{*}$ and $u^{*}(X)=$ $U^{*}-U_{>0}^{*}$. We describe the dynamic program for a slightly more general problem here: given bounds $A_{1}, A_{2}, B_{1}, B_{2}$, determine the cheapest subset $X$ satisfying $s^{*}(X) \in\left[A_{1}, A_{2}\right], u^{*}(X) \in\left[B_{1}, B_{2}\right]$. Such a dynamic program can be used for the current case by setting $A_{1}=A_{2}=S_{0}^{*}$ and $B_{1}=B_{2}=U^{*}-U_{>0}^{*}$, but will be useful when we consider the approximate version in the next section.

Let $\mathcal{S}\left(l, A_{1}, A_{2}, B_{1}, B_{2}\right)$ denote a subset of the links $\{1, \ldots, l\}$ which minimizes the cost

$$
C\left(\mathcal{S}\left(l, A_{1}, A_{2}, B_{1}, B_{2}\right)\right)=\sum_{i \in \mathcal{S}\left(l, A_{1}, A_{2}, B_{1}, B_{2}\right)} c_{i},
$$

while satisfying the two constraints

$$
s^{*}\left(\mathcal{S}\left(l, A_{1}, A_{2}, B_{1}, B_{2}\right)\right) \in\left[A_{1}, A_{2}\right] \quad \text { and } \quad u^{*}\left(\mathcal{S}\left(l, A_{1}, A_{2}, B_{1}, B_{2}\right)\right) \in\left[B_{1}, B_{2}\right] .
$$

The cost is 0 when $l=0$ and is defined to be $\infty$ if $\mathcal{S}()$ is empty, i.e, if no feasible subset exists. The recurrence equation is now defined as follows:

If

$$
C\left(\mathcal{S}\left(m-1, A_{1}-s_{m}^{*}, A_{2}-s_{m}^{*}, B_{1}-u_{m}^{*}, B_{2}-u_{m}^{*}\right)\right)+c_{m}<C\left(\mathcal{S}\left(m-1, A_{1}, A_{2}, B_{1}, B_{2}\right)\right),
$$

then

$$
\mathcal{S}\left(m, A_{1}, A_{2}, B_{1}, B_{2}\right)=\mathcal{S}\left(m-1, A_{1}-s_{m}^{*}, A_{2}-s_{m}^{*}, B_{1}-u_{m}^{*}, B_{2}-u_{m}^{*}\right) \cup\{m\}
$$


else

$$
\mathcal{S}\left(m, A_{1}, A_{2}, B_{1}, B_{2}\right)=\mathcal{S}\left(m-1, A_{1}, A_{2}, B_{1}, B_{2}\right)
$$

The former is relevant if $m$ is chosen to be in the subset, and the latter if $m$ is not). This immediately suggests the dynamic program, with a total storage of at most $m \cdot S_{0}^{*} \cdot U_{0}^{*}$, which is pseudopolynomial.

\subsection{Finding an Approximate Solution}

In the previous section, we showed that if we knew the invariants $L^{*}, D^{*}, S_{0}^{*}$ exactly, we could compute the optimum stackelberg strategy. We cannot expect to know these quantities exactly, but can guess them within a factor of $1+\delta$, simply by trying all possible powers of $1+\delta$. If these quantities are polynomially bounded, the number of trials is bounded by a polynomial in $\frac{\log n}{\log (1+\delta)}$. We show now that with this slack, we can still obtain an approximate solution.

We assume here that all the latency functions are rational functions of polynomials with polynomially bounded integral coefficients and exponents. This allows us to estimate the assignments on the links, given the latencies on them (which we guess, as mentioned above) and also ensures that when the assignment on a link is increased by a small factor, the latency does not blow up. We will have a fixed parameter $\delta$, which depends on $\epsilon$, and another parameter, $\delta_{1}$, is chosen so that $\ell_{i}((1+2 \delta) x) \leq\left(1+\delta_{1}\right) \ell_{i}(x)$ for any $i, x$. For our purposes, $\delta$ will be chosen to be inverse polynomial.

Following the discussion above, assume that we have guessed $L, D, S, U_{>0}$ so that $L \in\left[L^{*},(1+\right.$ $\left.\left.\delta_{2}\right) L^{*}\right], D \in\left[D^{*},\left(1+\delta_{2}\right) D^{*}\right], S_{0} \in\left[S_{0}^{*},\left(1+\delta_{2}\right) S_{0}^{*}\right]$ and $U_{>0} \in\left[U_{>0}^{*},\left(1+\delta_{2}\right) U_{>0}^{*}\right]$ for a parameter $\delta_{2}$ to be specified below. For each link $i, s_{i}^{*}, u_{i}^{*}$ are defined as in the previous section. For each link $i$, solve for $\ell_{i}\left(x_{i}\right)=L$ and $\left(y_{i} \ell_{i}\left(y_{i}\right)\right)^{\prime}=D$ so that the estimates are at least as large as the exact roots of these equations, but not exceeding by a factor of $1+\delta_{2}$. By choosing $\delta_{2}<\delta$ appropriately, we can ensure that $u_{i}=x_{i}$ satisfies $u_{i} \in\left[u_{i}^{*},(1+\delta) u_{i}^{*}\right]$. If $\ell_{i}\left(y_{i}\right) \geq(1-\delta) L$, define $s_{i}=y_{i}{ }^{5}$, otherwise $s_{i}=\infty$. This gives us a tuple $\left(s_{i}, u_{i}\right)$ for each link $i$.

As before, $s_{i}$ is intended to be the assignment to link $i$ if it is in $M_{0}$ and $u_{i}$ is the assignment to link $i$ if it is in $M_{>0}$. The extra complication we will face is that even if $i \in M_{0}$, we may have $t_{i}>0$ in the approximate stackelberg solution we find.

The next lemma - a refinement of Lemma 3, shows how the problem of approximating the Stackelberg strategy can be viewed as an approximation to the knapsack problem.

Lemma 4 Let $X \subset E$ be a subset satisfying the following conditions.

1. $\sum_{i \in X} s_{i} \in\left[(1-\delta) S_{0}^{*},(1+\delta) S_{0}^{*}\right]$

2. $\sum_{i \notin X} u_{i} \in\left[(1-\delta)\left(r-S_{0}^{*}\right),(1+\delta)\left(r-S_{0}^{*}\right)\right]$

3. $X$ minimizes the cost $\sum_{i \in X} s_{i} \ell_{i}\left(s_{i}\right)$.

Consider the following stackelberg strategy s' induced by $X$ : if $s(X) \leq \alpha r /(1+2 \delta), s_{i}^{\prime}=(1+2 \delta) s_{i}, \forall i \in X$ and if $s(X)>\alpha r /(1+2 \delta), s_{i}^{\prime}=\frac{\alpha r}{s(X)} s_{i}$. Then, $C\left(s^{\prime}\right) \leq(1+\epsilon) C\left(s^{*}\right)$

The proof of Lemma 4 is based on the following proposition

\footnotetext{
${ }^{5}$ This ensures that if $s_{i}^{*}$ is finite and $\ell_{i}\left(s_{i}^{*}\right) \geq L^{*}, \ell_{i}\left(s_{i}\right) \geq(1-\delta) L^{*}$
} 
Proposition 1 Let $t^{\prime}$ be the Nash assignment induced by $s^{\prime}$ and $u^{\prime}=s^{\prime}+t^{\prime}$. Let $L^{\prime}$ be the common Nash latency on all edges $i$ such that $t_{i}>0$. Then the following hold:

1. $\forall i \in X, s_{i}^{\prime} \leq(1+2 \delta) s_{i}$.

2. $s^{\prime}(X) \geq S_{0}^{*}$.

3. $L^{\prime} \leq\left(1+\delta_{1}\right) L$.

Proof: The proof of Part 1 is Obvious.

\section{Part 2:}

If $s(X) \leq \alpha r /(1+2 \delta), s^{\prime}(X)=s(X)(1+2 \delta) \geq(1-\delta)(1+2 \delta) S_{0}^{*} \geq S_{0}^{*}$. Next, suppose $s(X)>$ $\alpha r /(1+2 \delta)$. By construction, $s^{\prime}(X)=\alpha r$. Now recall that $S_{0}^{*}$ is the fraction controlled by the stackelberg strategy, and is bounded by $\alpha r$, by definition.

\section{Part 3:}

To show this, we construct a Nash assignment $v$ on $\bar{X}$ such that $s^{\prime}(X)+v(\bar{X}) \geq r$, and the Nash latency $L_{v}$ induced by $v$ satisfies $L_{v} \leq\left(1+\delta_{1}\right) L$. Now, suppose $L^{\prime}>L_{v}$. This implies that $u_{i}^{\prime}>v_{i}, \forall i \in$ $\bar{X}$, which leads to a contradiction because $s(X)+u^{\prime}(\bar{X})$ would then exceed $r$. This leaves us with the specification of the assignment $v$. By Part $2, s^{\prime}(X) \geq S_{0}^{*}$. Define $v=\operatorname{Nash}(\bar{X}, u(\bar{X})(1+2 \delta))$. Since $u(\bar{X}) \geq(1-\delta)\left(r-S_{0}^{*}\right)$, we have $v(\bar{X}) \geq r-S_{0}^{*}$, and $s^{\prime}(X)+v(\bar{X}) \geq r$, the property we required above. Also, there exists $i \in \bar{X}$ such that $v_{i} \leq(1+2 \delta) u_{i}$, since $v(\bar{X})=(1+2 \delta) u(\bar{X})$, and this gives $L_{v}=\ell_{i}\left(v_{i}\right)=\ell_{i}\left((1+2 \delta) u_{i}\right) \leq\left(1+\delta_{1}\right) L$.

Proof of Lemma 4: As in Proposition 1, let $t^{\prime}$ be the Nash assignment induced by $s^{\prime}$ and $u^{\prime}=s^{\prime}+t^{\prime}$. Let $L^{\prime}$ be the common Nash latency on all edges $i$ such that $t>0$.

We bound the cost of $s^{\prime}+t^{\prime}$, by considering the cost over sets $X$ and $\bar{X}$ separately. First, consider set $\bar{X}$.

$$
C_{\bar{X}}\left(s^{\prime}\right)=\sum_{i \in \bar{X}} u_{i}^{\prime} L^{\prime}=u^{\prime}(\bar{X}) L^{\prime}
$$

Next, consider the cost restricted to set $X, \sum_{i \in X}\left(s_{i}^{\prime}+t_{i}^{\prime}\right) \ell_{i}\left(s_{i}^{\prime}+t_{i}^{\prime}\right)$. We argue in the following steps.

1. We first show that whenever $t_{i}^{\prime}>0, \ell_{i}\left(s_{i}^{\prime}+t_{i}^{\prime}\right)$ is close to $\ell_{i}\left(s_{i}^{\prime}\right)$.

2. Second, using this and the fact that $s_{i}^{\prime}$ is not much larger than $s_{i}$, we show that $\sum_{i} s_{i}^{\prime} \ell_{i}\left(s_{i}^{\prime}+t_{i}^{\prime}\right)$ is not much larger than $\sum_{i} s_{i} \ell_{i}\left(s_{i}\right)$ which in turn is close to $C_{M_{=0}}\left(s^{*}\right)$ because of the choice of set $X$.

3. This leaves us with the part $\sum_{i} t_{i}^{\prime} \ell_{i}\left(s_{i}^{\prime}+t_{i}^{\prime}\right)=t^{\prime}(X) L^{\prime}$. We will show that $t^{\prime}(X)+u^{\prime}(\bar{X})$ is not much bigger than $u(X)$, and this allows us to bound the sum of $t(X) L^{\prime}+u^{\prime}(\bar{X}) L^{\prime}$.

From Part 3 of Proposition 1, if $t_{i}^{\prime}>0$ for some $i \in X, \ell_{i}\left(s_{i}^{\prime}+t_{i}^{\prime}\right)=L^{\prime} \leq\left(1+\delta_{1}\right) L$. By construction,

$$
\forall i \in X, \ell_{i}\left(s_{i}\right) \geq(1-\delta) L \geq(1-\delta) L^{\prime} /\left(1+\delta_{1}\right)=(1-\delta) \ell_{i}\left(s_{i}^{\prime}+t_{i}^{\prime}\right) /\left(1+\delta_{1}\right),
$$

and using Part 1 of Proposition 1, we get

$$
\sum_{i \in X} s_{i}^{\prime} \ell_{i}\left(s_{i}^{\prime}+t_{i}^{\prime}\right) \leq(1+2 \delta)^{2}\left(1+\delta_{1}\right) \sum_{i \in X} s_{i} \ell_{i}\left(s_{i}\right) .
$$


Next, since $X$ is the cheapest set satisfying the feasibility conditions, we have

$$
\sum_{i \in X} s_{i} \ell_{i}\left(s_{i}\right) \leq \sum_{i \in M_{=0}} s_{i} \ell_{i}\left(s_{i}\right) \leq(1+\delta)\left(1+\delta_{1}\right) C_{M_{=0}}\left(s^{*}\right)
$$

Together, this gives us

$$
\sum_{i \in X} s_{i}^{\prime} \ell_{i}\left(s_{i}^{\prime}+t_{i}^{\prime}\right) \leq(1+2 \delta)^{3}\left(1+\delta_{1}\right)^{2} C_{M_{=0}}\left(s^{*}\right)
$$

Finally, we bound the part $t^{\prime}(X) L^{\prime}$. Note that $t^{\prime}(X)=r-s^{\prime}(X)-u^{\prime}(\bar{X}) \leq 2 \delta s^{\prime}(X)+(1+2 \delta) u(X)-u^{\prime}(X)$, because $s^{\prime}(X)+u(X) \geq(1-\delta) r$. Since $s^{\prime}(X) \leq s(X)(1+2 \delta)$ and $L^{\prime} \leq\left(1+\delta_{1}\right) L$, we have $s^{\prime}(X) L^{\prime} \leq$ $(1+2 \delta)\left(1+\delta_{1}\right) s(X)(1-\delta) L \leq(1+2 \delta)\left(1+\delta_{1}\right) \sum_{i \in X} s_{i} \ell_{i}\left(s_{i}\right) \leq(1+2 \delta)^{2}\left(1+\delta_{1}\right)^{2} C_{M_{=0}}\left(s^{*}\right)$, where the second inequality holds because $\ell_{i}\left(s_{i}\right) \geq(1-\delta) L, \forall i \in X$.

Putting all this together, we have

$$
\begin{aligned}
C\left(s^{\prime}\right) & =\sum_{i \in X}\left(s_{i}^{\prime}+t_{i}^{\prime}\right) \ell_{i}\left(s_{i}^{\prime}+t_{i}^{\prime}\right)+\sum_{i \in \bar{X}} u_{i}^{\prime} \ell_{i}\left(u_{i}^{\prime}\right) \\
& \leq(1+2 \delta)^{3}\left(1+\delta_{1}\right)^{2} C_{M_{=0}}\left(s^{*}\right)+2 \delta(1+2 \delta)^{2}\left(1+\delta_{1}\right) C_{M_{=0}}\left(s^{*}\right)+(1+2 \delta) u(\bar{X}) L^{\prime} \\
& \leq(1+4 \delta)^{3}\left(1+\delta_{1}\right)^{2} C_{M_{=0}}\left(s^{*}\right)+(1+2 \delta)\left(1+\delta_{1}\right) u(\bar{X}) L .
\end{aligned}
$$

Using the fact that $L \leq\left(1+\delta_{1}\right) L^{*}$ and that $u(\bar{X}) \leq(1+\delta)\left(r-S_{0}^{*}\right)$, we get $u(\bar{X}) L \leq(1+\delta)(1+$ $\left.\delta_{1}\right) C_{M_{>0}}\left(s^{*}\right)$. Therefore, $C\left(s^{\prime}\right) \leq(1+\epsilon) C\left(s^{*}\right)$, where $\epsilon$ is chosen so that $1+\epsilon=(1+4 \delta)^{3}\left(1+2 \delta_{1}\right)^{2}$.

Recall that we have estimates $S_{0} \in\left[S_{0}^{*},\left(1+\delta_{2}\right) S_{0}^{*}\right]$ and $U_{>0} \in\left[U_{>0}^{*},\left(1+\delta_{2}\right) U_{>0}^{*}\right]$ for appropriate $\delta_{2}<\delta$. Since we do not know $S_{0}^{*}, U_{>0}^{*}$ exactly, we will actually find the cheapest subset $X$ such that $s(X) \in\left[\left(1-\delta_{2}\right) S_{0},\left(1+\delta_{2}\right) S_{0}\right] \subset\left[(1-\delta) S_{0}^{*},(1+\delta) S_{0}^{*}\right]$ and $u(X) \in\left[U-\left(1+\delta_{2}\right) U_{>0}, U-\left(1-\delta_{2}\right) U_{>0}\right] \subset$ $\left[U-(1+\delta) U_{>0}^{*}, U-(1-\delta) U_{>0}^{*}\right]$ (which automatically ensures that $u(\bar{X}) \in\left[(1-\delta) U_{>0}^{*},(1+\delta) U_{>0}^{*}\right]$ ). This leaves us with the problem of finding an approximate solution and this is solved in the following steps.

1. Scaling Let $m_{s}=\max _{i}\left\{s_{i}: s_{i}<\infty\right\}$ and $m_{u}=\max _{i}\left\{u_{i}\right\}$. Define $\hat{s}_{i}=\left\lfloor\frac{s_{i} m}{\gamma m_{s}}\right\rfloor, \hat{u}_{i}=\left\lfloor\frac{u_{i} m}{\gamma m_{u}}\right\rfloor$, $\hat{S}_{0}=\left\lfloor\frac{S_{0} m}{\gamma m_{s}}\right\rfloor$ and $\hat{U}_{>0}=\left\lfloor\frac{U_{>0} m}{\gamma m_{u}}\right\rfloor$. Let $\hat{U}=\sum_{i} \hat{u}_{i}$. If $s_{i}>S_{0}, s_{i}<\infty$ for some $i$, it is clear that $i \in M_{>0}$, and we can remove link $i$ from consideration. Therefore, wlog we can assume that $m_{s} \leq S_{0}$.

Similarly, we can assume that $m_{u} \leq U_{>0}$.

2. The Dynamic Program Run the same dynamic program described in the preceeding section: compute the cheapest set $\mathcal{S}\left(m,\left(1-\delta_{3}\right) \hat{S}_{0},\left(1+\delta_{3}\right) \hat{S}_{0}, \hat{U}-\left(1+\delta_{3}\right) \hat{U}_{>0}, \hat{U}-\left(1-\delta_{3}\right) \hat{U}_{>0}\right)$, in the notation of the previous section, where $\delta_{3}$ is a small enough parameter to be fixed later. This gives us a set $X$ such that

$$
\left.\hat{s}(X) \in\left[\left(1-\delta_{3}\right) \hat{S}_{0},\left(1+\delta_{3}\right) \hat{S}_{0}\right], \quad \text { and } \quad \hat{u}(X) \in\left[\hat{U}-\left(1+\delta_{3}\right) \hat{U}_{>0}, \hat{U}-\left(1-\delta_{3}\right) \hat{U}_{>0}\right)\right]
$$

and the cost of $X$ is minimized. The running time of this step is $O\left(m^{5} / \gamma\right)$.

3. Retrieve a solution We claim that $X$ satisfies the original requirements.

First, we obtain bounds on $s(X)$. By construction, it follows that

$$
s(X) \leq \gamma m_{s}(\hat{s}(X)+|X|) / m \leq\left(1+\delta_{3}\right) S_{0}+\gamma m_{s} \leq\left(1+\delta_{3}+\gamma\right) S_{0} .
$$


Also,

$$
\begin{aligned}
s(X) & \geq \gamma m_{s} \hat{s}(X) / m \\
& \geq \frac{\gamma m_{s}}{m}\left(1-\delta_{3}\right) \hat{S}_{0} \\
& \geq \frac{\gamma m_{s}}{m}\left(1-\delta_{3}\right)\left(\frac{S_{0} m}{\gamma m_{s}}-1\right) \\
& \geq\left(1-\delta_{3}\right) S_{0}-\left(1-\delta_{3}\right) \frac{\gamma m_{s}}{m} \\
& \geq\left(1-\delta_{3}\right) S_{0}-\left(1-\delta_{3}\right) \gamma S_{0} \\
& \geq\left(1-\delta_{3}\right)(1-\gamma) S_{0}
\end{aligned}
$$

Choose $\delta_{2}$ so that

$$
\delta_{3}+\gamma \leq \delta_{2} \quad \text { and } \quad\left(1-\delta_{3}\right)(1-\gamma) \geq\left(1-\delta_{2}\right),
$$

then we get $s(X) \in\left[\left(1-\delta_{2}\right) S_{0},\left(1+\delta_{2}\right) S_{0}\right]$.

Next, we bound $u(X)$. By construction, we can upper bound $u(X)$ in the following way.

$$
\begin{aligned}
u(X) & \leq \frac{\gamma m_{u}}{m}(\hat{u}(X)+|X|) \\
& \leq \frac{\gamma m_{u}|X|}{m}+\frac{\gamma m_{u}}{m}\left(\hat{U}-\left(1-\delta_{3}\right) \hat{U}_{>0}\right) \\
& \leq \gamma m_{u}+U-\frac{\left(1-\delta_{3}\right) \gamma m_{u}}{m}\left(\frac{U_{>0} m}{\gamma m_{u}}-1\right) \\
& \leq U-\left(1-\delta_{3}\right) U_{>0}+\gamma m_{u}+\left(1-\delta_{3}\right) \gamma m_{u} \\
& \leq U-\left(1-\delta_{3}-2 \gamma\right) U_{>0} .
\end{aligned}
$$

Finally, we obtain a lower bound for $u(X)$ :

$$
\begin{aligned}
u(X) & \geq \frac{\gamma m_{u}}{m} \hat{u}(X) \\
& \geq \frac{\gamma m_{u}}{m}\left(\hat{U}-\left(1+\delta_{3}\right) \hat{U}_{>0}\right) \\
& \geq \frac{\gamma m_{u}}{m}\left(\frac{U m}{\gamma m_{u}}-m-\left(1+\delta_{3}\right) \hat{U}_{>0}\right) \\
& \geq U-\gamma m_{u}-\left(1+\delta_{3}\right) U_{>0} \\
& \geq U-\left(1+\delta_{3}+\gamma\right) U_{>0} .
\end{aligned}
$$

If $\delta_{2}$ satisfies $\delta_{3}+2 \gamma \leq \delta_{2}$ we get

$$
u(X) \in\left[U-\left(1+\delta_{2}\right) U_{>0}, U-\left(1-\delta_{2}\right) U_{>0}\right] .
$$

The quantities $\delta_{2}, \delta_{3}, \gamma$ can be chosen so that all the above constraints are satisfied.

\section{Extension to Layered Graphs with Bounded Width}

Consider a layered graph $G$ with layers $V_{0}, V_{1}, \ldots, V_{k}$ of vertices. All edges go from $V_{i}$ to $V_{i+1}$ for some $i$, and $u, v$ are the source and sink, with $u \in V_{0}, v \in V_{k}$. Assume that $\left|V_{i}\right| \leq w, \forall i$. Again, a total of $r$ units of 
flow has to be sent from $s$ to $t$, and the stackelberg strategy can control $\alpha r$ part of this. A stackelberg strategy now corresponds to deciding $u \rightarrow v$ flow paths, with a total of $\alpha r$ flow on them. Corresponding to a given stackelberg strategy $s$, we have a Nash flow $t$ consisting of flow paths. The Nash flow satisfies the property $\ell_{P}(t+s)=\ell_{P^{\prime}}(t+s)$ for every pair of paths $P, P^{\prime}$ such that $t_{P}, t_{P^{\prime}}>0$. We show that there is a simple dynamic programming solution to approximate the stackelberg strategy when the latency functions are all polynomial functions.

With each layer $V_{i}$, we associate a vector $h=<h_{x}, x \in V_{i}>$, which we call the flow vector. For each vertex $x, h_{x}$ is a triplet $h_{x}=\left(L_{x}, r_{x}, \alpha_{x}\right)$, whose semantics are described below. The quantity $r_{x}$ denotes the total flow between $x$ and $v$ carried by $s+t$, and $\alpha_{x}$ is the fraction of flow carried by stackelberg paths. All Nash flow paths from $u$ to $v$ passing through vertex $x$ can be decomposed into paths from $u$ to $x$ and from $x$ to $v$. The latency on the path segments from $x$ to $v$ is a constant, which we call the common Nash latency, and denote by $L_{x}$ above. We will also let $L_{x}$ be undefined, if no Nash flow path passes through $x$. Such a vector $h$ for layer $V_{i}$ completely captures the state of the flow through vertices in $V_{i}$. Though we would not actually know the correct quantities, we shall guess them within a $1+\delta$ factor. The optimum $s+t$ flow from $u$ to $v$ inducing a corresponding vector $h$ on each layer $V_{i}$ and the dynamic program basically finds the best flow when the flow vectors at each layer are rounded to a power of $1+\delta$. The total cost of $s+t$ is the sum of the costs of the flow from layer $V_{i}$ to $V_{i+1}$, over all $i$. Therefore, the problem of finding the best flow reduces to that of finding the cheapest flow from layer $V_{i}$ to $V_{i+1}$, for given flow vectors on $V_{i}, V_{i+1}$. This immediately suggests the dynamic program for computing an approximation to the best $s+t$ flow. Starting from $i=k$, we compute the best $s+t$ flow from $V_{i+1}$ to $v$, for each possible flow vector $h$ on $V_{i+1}$. Next, for any flow vector $h^{\prime}$ on $V_{i}$, we find the cheapest flow from $V_{i}$ to $V_{i+1}$, for each flow vector $h$ on $V_{i+1}$. This flow is the cheapest flow from $V_{i}$ to $v$, with a flow vector $h^{\prime}$ on $V_{i}$. Of course, we are only going to solve the subproblems for values of $L_{x}, r_{x}, \alpha_{x}, \forall x$, which are powers of $1+\delta$. If the largest of these values is $N$, the number of flow vectors at any layer is bounded by $\left(\frac{\log N}{\log 1+\delta}\right)^{3 w}$, which is polynomial if $N$ is polynomial.

We now consider the subproblem of finding the cheapest flow from $V_{i}$ to $V_{i+1}$, given flow vectors $h, h^{\prime}$ on $V_{i}, V_{i+1}$ respectively. The other remaining issue of bounding the total error is addressed later. Let $\left(I_{x}, r_{x}, \alpha_{x}\right)$ denote the triplet corresponding to vertex $x \in V_{i} \cup V_{i+1}$ in $h, h^{\prime}$. First, consider the case where there are no parallel edges; this restriction is removed a little later. Since there are only $\imath^{2}$ edges, guess a subset $E$ of edges which carry positive Nash flow (we will try out every possible subset $E$, resulting in $2^{w^{2}}$ iterations). For each edge $e=\left(w, w^{\prime}\right) \in E^{\prime}, L_{w}, L_{w^{\prime}}$ must be defined and $L_{w} \geq L_{w^{\prime}}$ (if not, $E^{\prime}$ is not a valid guess), and this determines the flow $f_{e}$ on edge $e$ such that $\ell_{e}\left(f_{e}\right)=L_{w}-L_{w^{\prime}}$. This allows us to formulate the following flow problem, of similar nature as that of [RT00]. We have variables $s_{e}, t_{e}$ on each edge, specifying the stackelberg and Nash flows. One problem is that since the flow vectors $h, h$ are all aproximate, there may be no flow that satisfies the feasibility constraints exactly. Therefore, we will relax the feasibility constraints set by $h$ on $V_{i}$. 
Then, the problem is to minimize $\sum_{e} s_{e} \ell_{e}\left(s_{e}\right)$ subject to

$$
\begin{aligned}
& \sum_{e=\left(w, w^{\prime}\right), \forall w^{\prime}} s_{e} \in\left[(1-\delta) \alpha_{w} r,(1+\delta) \alpha_{w} r\right] \quad \forall w \in V_{i} \\
& \sum_{e=\left(w, w^{\prime}\right), \forall w} s_{e}=\alpha_{w^{\prime}} r \quad \forall w^{\prime} \in V_{i+1} \\
& \sum_{e=\left(w, w^{\prime}\right), \forall w^{\prime}}\left(s_{e}+t_{e}\right) \in\left[(1-\delta) r_{w},(1+\delta) r_{w}\right] \quad \forall w \in V_{i} \\
& \sum_{e=\left(w, w^{\prime}\right), \forall w}\left(s_{e}+t_{e}\right)=r_{w^{\prime}} \quad \forall w^{\prime} \in V_{i+1} \\
& s_{e}+t_{e} \in\left[(1-\delta) f_{e},(1+\delta) f_{e}\right] \quad \forall e \in E^{\prime}
\end{aligned}
$$

This problem can be solved by the methods described in [RT00] because we still have a convex program. For each choice of $h^{\prime}$ on $V_{i+1}$, compute the cheapest possible flow and the cheapest such flow, which is combined with the current flow assignment between layers $V$ and $V_{i+1}$ to get the solution for flow vector $h$ on $V_{i}$. Notice that since the flow constraints on $V_{i}$ are satisfied approximately, this flow induces a vector $\hat{h}$ on $V_{i}$, in which every entry is within a $1 \pm \delta$ factor of the corresponding entry in $h$.

Because of this problem, the dynamic program is actually more complicated. For layer $V_{+1}$, we have a set $F_{i+1}$ of possible flow vectors, and for each $h \in F_{i+1}$, there is a vector $\hat{h}$ close enough to $h$, having a realizable flow starting at $h$. Now when we are computing from layer $V_{i}$, we choose a vector $g \in F_{i}$, and for each $h \in F_{i+1}$, we solve for the flow when the flow vectors are $g$ on $V_{i}$ and $\hat{h}$ on $V_{i+1}$, find the cheapest such flow over all $h$, and find the actual $\hat{g}$ induced on $V_{i}$.

Finally, we show that the total error is bounded, by the following series of lemmas. Suppose the optimum flow induces flow vectors $h_{o p t}, h_{\text {opt }}^{\prime}$ on layers $V_{i}, V_{i+1}$. The dynamic program will look for the flow with $h, h^{\prime}$ which are within a $1+\delta$ factor of $h_{o p t}, h_{o p t}^{\prime}$. Let $s_{o p t}+t_{o p t}, s+t$ be the flow assignments between layers $V_{i}, V_{i+1}$ in the optimum and the approximate solution, respectively.

Lemma 5 Let $s_{\text {opt }}+t_{\text {opt }}, s+t$ be defined as above. Then, $C(s+t) \leq(1+\epsilon) C\left(s_{\text {opt }}+t_{\text {opt }}\right)$.

The proof of this lemma relies on the facts that the edges have no capacities and small perturbations in the flow on the edges do not change the cost by much. This allows us to show that the net flow induced by $s+t$ is close to that induced by $s_{o p t}+t_{\text {opt }}$ on each edge. This lemma allows us to bound the cost of $s+t$ with respect to the optimum.

One problem is that the final $t$ we compute by combining the assignments across the layers need not be the actual nash flow, but is an approximate one: some nash flow paths could exceed others in latency by a factor of $1+\epsilon$. Let $t^{\prime}$ be the actual nash flow induced by $s$.

Lemma 6 Let $s, t, t^{\prime}$ be as defined above and $L(t), L\left(t^{\prime}\right)$ be the common nash flow latencies of $t$ and $t$, respectively. Then, $L\left(t^{\prime}\right) \leq(1+\epsilon) L(t)$.

\section{Two-round Stackelberg Strategy}

Denote the stackelberg strategy considered in the previous section by 1SS. We consider below a two round modification of this strategy, called 2SS, and denoted by $(\mathbf{s}, \mathbf{s})$. 
1. Choose a strategy $\mathbf{s}=<s_{1}, \ldots, s_{m}>$ satisfying $\sum_{i} s_{i} \leq \alpha r$

2. Let $\mathbf{t}$ be the Nash-equilibrium induced by $\mathbf{s}$.

3. Keep $\mathbf{t}$ fixed and change $\mathbf{s}$ to vector $\mathbf{s}$

The goal is to choose $\mathbf{s}$ and $\mathbf{s}^{\prime}$ so that $\mathbf{s}^{\prime}+\mathbf{t}$ has as small a cost as possible, compared to the optimum. By $C\left(\mathbf{s}, \mathbf{s}^{\prime}\right)$, we denote the cost $C\left(\mathbf{s}^{\prime}+\mathbf{t}\right)$ of the assignment resulting from the two-round stackelberg strategy $\left(\mathbf{s}, \mathbf{s}^{\prime}\right)$. The one-round stackelberg strategy leads to an assignment with cost at most $\frac{1}{\alpha}$ times the optimal, and so the question is whether a two-round strategy leads to a constant factor improvement.

It is easy to see that further rounds do not help. If we have $k$ alternating stackelberg/Nash strategy, the final solution just depends on the final round. Surprisingly, if the leader plays after the remaining players have formed a Nash equilibrium, the resulting solution is at least as good as 1SS.

\subsection{The quality of $2 \mathrm{SS}$}

While 2SS might not guarantee a factor better than 1SS, we show that it is quite often much better. In what follows, let $\mathbf{x}=\operatorname{OPT}(S, r)$ and $\mathbf{y}=\operatorname{Nash}(S, r)$ where $S \subset M, M$ being the set of all links. If $S$ is understood or if $S=M$, we sometimes just write $O P T(r)$ and $N a \operatorname{sh}(r)$. Let $A=\left\{i: x_{i} \geq y_{i}\right\}$. Assume that the links are ordered in such a way that $\ell_{m}\left(x_{m}\right) \geq \ldots \geq \ell_{1}\left(x_{1}\right)$. If $x(A)-y(A) \geq \alpha r$, the best that 1SS can guarantee is $\frac{1}{\alpha}$, which is achieved by the Nash solution itself. The factor guaranteed asymptotically by $2 \mathrm{SS}$ in this case is also $\frac{1}{\alpha}$, though it does better in a large class of instances. On the other hand, if $x(A)-y(A)<\alpha, 2$ SS always gives an optimal solution, while 1SS could still give a factor of $\frac{1}{\alpha}$ in the worst case. We show an example where both 1SS and 2SS are just as expensive as the nash solution. We then describe some classes of instances where 2SS gives a provably better guarranty.

As described earlier, both 1SS and 2SS could be as bad as the Nash solution in some cases.

Lemma 7 Let $\boldsymbol{x}=O P T(r)$ and $\boldsymbol{y}=N a s h(r)$. Let $A=\left\{i: x_{i} \geq y_{i}\right\}$. If $x(A)-y(A) \geq \alpha r, C(\boldsymbol{y}) \leq$ $\frac{1}{\alpha} C(\boldsymbol{x})$. If $x(A)-y(A)<\alpha, 2 S S$ leads to the optimum solution.

Proof: Let $L(y)$ be the common Nash latency. Assume first that $x(A)-y(A) \geq \alpha r$. Then, $C\left(x_{A}\right) \geq$ $(\alpha r+y(A)) L(y)$. Therefore, $C(y)=r L(y) \leq \frac{1}{\alpha+y(A) / r} C(x)$. Next, consider the case $x(A)-y(A)<\alpha r$. Choose the vector $s$ to be $s_{i}=y_{i}-x_{i}, i \notin A$ and $s_{i}=0, i \in A$. Then $\sum_{i} s_{i} \leq \alpha r$. The induced Nash equilibrium will then be $t_{i}=y_{i}-s_{i}, \forall i$. In the second round, choose $s_{i}^{\prime}=x_{i}-y_{i}, i \in A$ and $s_{i}^{\prime}=0, i \notin A$. Then $\sum_{i} s_{i}^{\prime}=\sum_{i} s_{i}$ and $s^{\prime}+t$ gives exactly the optimum solution $x$.

Note that the above scheme for 2SS actually results in a factor of at most $\frac{1}{\alpha+y(A) / r}$. The LLF strategy for 1SS only guarantees a factor of $\frac{1}{\alpha}$ and we believe no strategy for 1SS can actually do better.

A tight example We describe an instance below where the factor $\frac{1}{\alpha}$ is tight. Consider a graph $G$ with two nodes $u, v$ and two parallel edges $e, f$ between $u, v$. Define the latency functions $\ell_{e}, \ell_{f}$ are shown in Figure ??. Assume that $\delta_{1}<\delta_{2}$ and $\epsilon, \delta_{1}, \delta_{2}$ are all very small quantities.

In this example, the optimum assignment assigns slightly less than $\alpha+\delta_{2}$ on link $e$ and slightly more than $1-\alpha-\delta_{2}$ on link $f$, leading to a total cost of almost $\left(\alpha+\delta_{2}\right)(1+\epsilon) L$. The nash equilibrium is $\left(\delta_{1}, 1-\delta_{1}\right)$, 


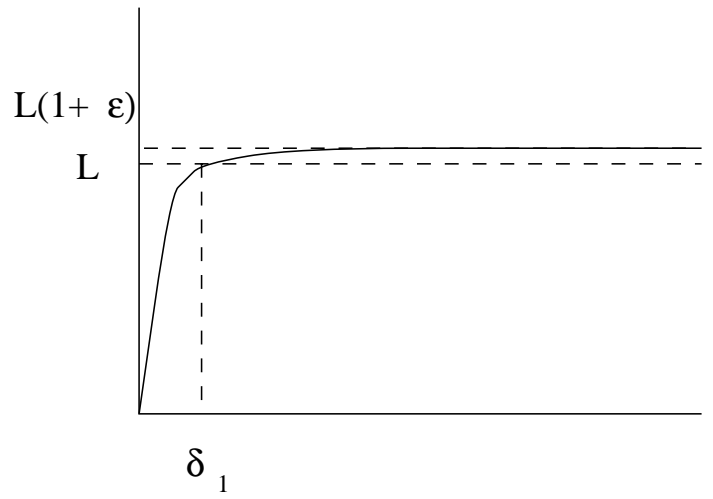

(a)

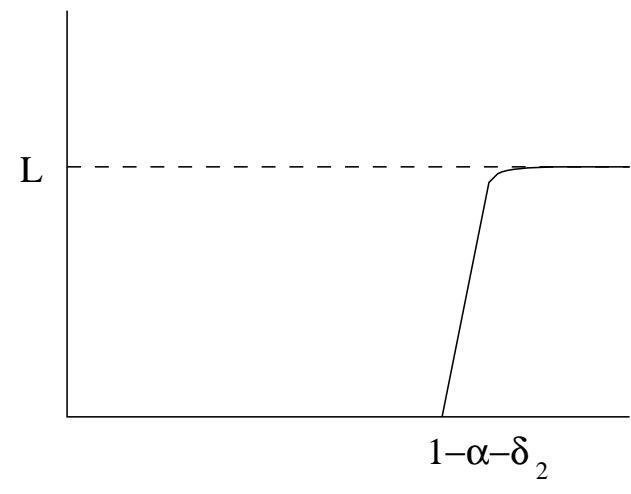

(b)

Figure 1: A tight example:(a) function $\ell_{e},(b)$ function $\ell_{f}$

with a total cost of $L$. It is easy to see that any 1SS strategy that controls at most $\alpha$ amount does not improve on the cost of nash. 2SS does not improve the cost either, since $\delta<\delta_{2}$. The basic problem in this example is that the latency functions could increase sharply. When their slopes are bounded, the following lemmas show that one can obtain better bounds.

The worst case bounds can be improved when the latency functions are restricted. If $\ell(z(1+\delta)) \geq$ $\phi(\delta) \ell_{i}(z)$ for each $i$, the guarantee achieved by 2SS can be improved. Such an assumption is not too unrealistic, since functions growing as fast as a polynomial have this property at least asymptotically.

Lemma 8 Let $\forall i, u \quad \ell_{i}(u(1+\delta)) \geq \phi(\delta) \ell_{i}(u)$. Then if $\alpha<1$ and $\phi\left(\frac{1}{1-\alpha}\right)>1$, then there exists a $2 S S$ strategy, $\left(\mathbf{s}, \mathbf{s}^{\prime}\right)$, such that

$$
C\left(\mathbf{s}, \mathbf{s}^{\prime}\right) \leq \frac{1+\alpha \phi\left(\frac{1}{1-\alpha}\right)}{\alpha \phi\left(\frac{1}{1-\alpha}\right)} C(\boldsymbol{x})<\frac{1}{\alpha} C(\boldsymbol{x}) .
$$

Proof: We can assume that $x(A)-y(A) \geq \alpha r$, else the previous lemmas show that 2SS gives the optimal solution. Let $\hat{\mathbf{z}}=\operatorname{Nash}(\bar{A},(1-\alpha) r-y(A))$. Define the stackelberg strategy $\mathbf{s}$ in the first round as $s_{i}=y_{i}-\hat{z}_{i}, i \in \bar{A}$ and $s_{i}=0, i \in A$. Then $\sum_{i} s_{i}=\alpha r$ and the induced Nash equilibrium, $\mathbf{t}$ is such that $\mathbf{s}+\mathbf{t}=\mathbf{y}$. In the second round, choose any $\mathbf{s}$ such that $s_{i}^{\prime}=0, i \in \bar{A}$ and $y_{i} \leq s_{i}^{\prime} \leq x_{i}, i \in A$ and $\sum_{i} s_{i}^{\prime}=\alpha r$. Let $\mathbf{z}=\mathbf{s}^{\prime}+\mathbf{t}$. Clearly, $z_{i}=\hat{z}_{i}, i \in \bar{A}$ and $\frac{y(\bar{A})}{z(\bar{A})}=\frac{1-y(A) / r}{1-\alpha-y(A) / r} \geq \frac{1}{1-\alpha}$. Therefore, there is an $i \in \bar{A}$ such that $y_{i} \leq z_{i} /(1-\alpha)$. From our assumption about the latency functions, $\ell_{i}\left(y_{i}\right) \geq \ell_{i}\left(z_{i}\right) \phi\left(\frac{1}{1-\alpha}\right)$. Let $L(\mathbf{y})$ be the common Nash latency for $y$ and $L(\mathbf{z})$ be the common latency of $z$, on $\bar{A}$.

Now,

$$
C\left(\mathbf{x}_{A}\right) \geq C\left(\mathbf{z}_{A}\right)=(y(A)+\alpha) L(\mathbf{y}) \geq\left(y(A)+\alpha \phi\left(\frac{1}{1-\alpha}\right)\right) L(\mathbf{z}) .
$$

$C\left(\mathbf{z}_{\bar{A}}\right)=(1-\alpha-y(A)) L(\mathbf{z})$. This gives us

$$
(1-y(A)-\alpha) C\left(\mathbf{z}_{A}\right) \geq\left(y(A)+\alpha \phi\left(\frac{1}{1-\alpha}\right)\right) C\left(\mathbf{z}_{\bar{A}}\right),
$$

which implies

$$
C(\mathbf{x}) \geq C\left(\mathbf{z}_{A}\right) \geq \frac{y(A)+\alpha \phi}{1+\alpha(\phi-1)} C(\mathbf{z})
$$


where $\phi=\phi\left(\frac{1}{1-\alpha}\right)$. Therefore,

$$
C(\mathbf{z}) / C(\mathbf{x}) \leq \frac{1+\alpha\left(\phi\left(\frac{1}{1-\alpha}\right)-1\right)}{\alpha \phi\left(\frac{1}{1-\alpha}\right)}
$$

\subsection{Linear Latency Functions}

Roughgarden[Ro01] shows that the LLF strategy for 1SS yields an assignment of cost bounded by $\frac{4}{3+\alpha}$ times the optimal, when the latency functions are all linear. We show that 2SS gives a strictly better bound for this case. In this section, we assume that the latency function for link $i$ has the form $\ell(u)=a_{i} u+b_{i}, i=$ $1, \ldots, m$ and $a_{i}, b_{i} \geq 0, \forall i$.

The following lemma relates the costs of the optimum flow of $r$ and the nash flow of $r$.

Lemma 9 Suppose $\mathbf{x}=O P T(S, r)$ and $\mathbf{y}=\operatorname{Nash}\left(S, r^{\prime}\right)$. Then, $C(\mathbf{x})=\left(r-\frac{r^{\prime}}{4}\right) L(\mathbf{y})+\sum_{i} a_{i}\left(x_{i}-\right.$ $\left.\frac{y_{i}}{2}\right)^{2}+\frac{b_{i} y_{i}}{4}$, where $L(\mathbf{y})$ is the common Nash latency for $\mathbf{y}$.

Proof:

$$
\begin{aligned}
C(\mathbf{x}) & =\sum_{i} a_{i} x_{i}^{2}+b_{i} x_{i} \\
& =\sum_{i}\left(x_{i}-y_{i} / 4\right)\left(a_{i} y_{i}+b_{i}\right)+\sum_{i} a_{i}\left(x_{i}-y_{i} / 2\right)^{2}+b_{i} y_{i} / 4 \\
& =\sum_{i}\left(x_{i}-y_{i} / 4\right) L(\mathbf{y})+\sum_{i} a_{i}\left(x_{i}-y_{i} / 2\right)^{2}+b_{i} y_{i} / 4 \\
& =\left(r-r^{\prime} / 4\right) L(\mathbf{y})+\sum_{i} a_{i}\left(x_{i}-y_{i} / 2\right)^{2}+b_{i} y_{i} / 4
\end{aligned}
$$

A Reassignment Operation The 2SS strategy we consider later involves incrementing the assignment on a set of links, $A$, that already has a nash assignment. We describe the operation here, and bound the cost after the increase. Let $A=\{1, \ldots, a\}$ be a set of links. Let $\mathbf{x}=O P T(A, \beta)$ and $\mathbf{y}=\operatorname{Nash}(A, \boldsymbol{\beta})$ with $\beta \geq \beta^{\prime}+\alpha$. We will always be considering situations where $L(\mathbf{y}) \leq \ell_{1}\left(x_{1}\right) \leq \ldots \leq \ell_{a}\left(x_{a}\right)$, where $L(\mathbf{y})=\ell_{i}\left(y_{i}\right), \forall i \in A$ is the common nash latency induced by $\mathbf{y}$. Our goal is to add a total of $\alpha$ amount to $\mathbf{y}$, while keeping the cost bounded. Let $d \leq a$ be the smallest index satisfying the following properties.

1. $\sum_{i \leq a^{\prime}} x_{i}-y_{i} \leq \alpha$

2. $x_{i} \geq \hat{z}_{i}, a^{\prime}+1 \leq i \leq a$, where $\hat{\mathbf{z}}=\operatorname{Nash}\left(\left\{a^{\prime}+1, \ldots, a\right\}, \beta^{\prime}+\alpha-\sum_{i \leq a^{\prime}} x_{i}\right)$

3. $\ell_{a^{\prime}}\left(x_{a^{\prime}}\right) \leq L(\hat{\mathbf{z}})$, where $L(\hat{\mathbf{z}})=\ell_{i}\left(\hat{z}_{i}\right)$ is the common nash latency of $\hat{\mathbf{z}}$.

It is easy to see that such an $d^{\prime}$ exists. Denote the flow on $A$ by a variable $\theta$, and think of increasing it continuously, while maintaining a nash assignment, denoted by $y$. At some point, when $\theta=\theta_{1}$, we will have $\ell_{1}\left(y_{1}^{\theta}\right)=\ell_{1}\left(x_{1}\right)$. From this instant, keep the assignment on link 1 fixed, and increase the flow on the 
remaining links, and so on. This process stops at the index $d$ defined above. Define $A_{1}=\left\{1, \ldots, a^{\prime}\right\}, A_{2}=$ $A \backslash A_{1}, x\left(A_{1}\right)=\beta_{1}$. Let $\mathbf{s}$ ' be an assigment such that $s_{i}^{\prime}=x_{i}-y_{i}, \forall i \in A_{1}$ and $s_{i}^{\prime}=\hat{z}_{i}-y_{i}, \forall i \in A_{2}$ and let $\mathbf{y}+\mathbf{s}^{\prime}=\mathbf{z}$. Note that $\sum_{i} s_{i}^{\prime}=\alpha$. Later, we will refer to this step as a reassignment operation, which adds the vector s' defined above to the assignment $\mathbf{y}$ on set $A$.

Observation 2 Let $\boldsymbol{x}, \boldsymbol{z}, A$ be defined as above. Then $C\left(\boldsymbol{x}_{A}\right) \geq \frac{\beta}{\beta^{\prime}+\alpha} C\left(\boldsymbol{z}_{A}\right)$.

Proof: $C\left(\mathbf{x}_{A_{2}}\right)=\left(\beta^{\prime}+\alpha-\beta_{1}\right) L$, where $L$ is the common Nash latency of $\mathbf{z}$ on $A_{2}$. Since $\forall i \in A_{2}, \ell_{i}\left(x_{i}\right) \geq$ $\ell_{i}\left(z_{i}\right)$

$$
C\left(\mathbf{x}_{A_{2}}\right) \geq \sum_{i \in A_{2}} x_{i} L=\frac{\beta-\beta_{1}}{\beta^{\prime}+\alpha-\beta_{1}} C\left(\mathbf{z}_{A_{2}}\right) .
$$

This implies

$$
\left(\beta^{\prime}+\alpha\right) C\left(\mathbf{x}_{A_{2}}\right) \geq \beta C\left(\mathbf{z}_{A_{2}}\right)+\beta_{1}\left(C\left(\mathbf{x}_{A_{2}}\right)-C\left(\mathbf{z}_{A_{2}}\right)\right) \geq \beta C_{A_{2}}(z)+\beta_{1}\left(\beta-\beta^{\prime}-\alpha\right) L .
$$

Then since $C\left(\mathbf{x}_{A_{1}}\right)=C\left(\mathbf{z}_{A_{1}}\right), \quad \forall i \in A_{1}, \ell_{i}\left(x_{i}\right) \leq L$, and $x\left(A_{1}\right)=\beta_{1}$, we get

$$
\left(\beta^{\prime}+\alpha\right) C\left(\mathbf{x}_{A_{1}}\right)=\beta C\left(\mathbf{z}_{A_{1}}\right)-\left(\beta-\beta^{\prime}-\alpha\right) C\left(\mathbf{z}_{A_{1}}\right) \geq \beta C\left(\mathbf{z}_{A_{1}}\right)-\left(\beta-\beta^{\prime}-\alpha\right) \beta_{1} L .
$$

Adding up these inequalities, we have $(\beta+\alpha) C(\mathbf{x}) \geq \beta C(\mathbf{z})$.

As in [Ro01], we order the links so that $b_{1} \leq \ldots \leq b_{m}$, and we can assume that $a_{i}=0$ for at most one link $i$, which can be assumed to be the last one, if it exists. We recall the following lemmas from [Ro01].

Lemma 10 (lemma 5.1 in [Ro01]) The nash assignment $\mathbf{y}$ is given by

$$
\mathbf{y}=\sum_{i=1}^{m} \delta_{i} \frac{\mathbf{v}_{\mathbf{i}}}{\left\|\mathbf{v}_{\mathbf{i}}\right\|},
$$

where $\mathbf{v}_{\mathbf{i}}$ is the vector $\left(\frac{1}{a_{1}}, \ldots, \frac{1}{a_{m}}\right)$ and $\delta_{i}$ is defined inductively as: $\delta_{0}=0, \delta_{i}=\min \left\{\left(b_{i+1}-b_{i}\right)\left\|\mathbf{v}_{\mathbf{i}}\right\|\right.$ ,$\left.r-\sum_{j=0}^{i-1} \delta_{j}\right\}$ and $\delta_{m}=r-\sum_{j=0}^{m} \delta_{j}$.

Lemma 11 (lemma 5.2 in [RoO1]) The optimal assignment $\mathbf{x}$ is given by

$$
\mathbf{x}=\sum_{i=1}^{m} \delta_{i}^{*} \frac{\mathbf{v}_{\mathbf{i}}}{\left\|\mathbf{v}_{\mathbf{i}}\right\|}
$$

where $\mathbf{v}_{\mathbf{i}}$ is the vector $\left(\frac{1}{a_{1}}, \ldots, \frac{1}{a_{m}}\right)$ and $\delta_{i}^{*}$ is defined inductively as: $\delta_{0}^{*}=0, \delta_{i}^{*}=\min \left\{\frac{1}{2}\left(b_{i+1}-b_{i}\right)\left\|\mathbf{v}_{\mathbf{i}}\right\|\right.$ ,$\left.r-\sum_{j=0}^{i-1} \delta_{j}\right\}$ and $\delta_{m}^{*}=r-\sum_{j=0}^{m} \delta_{j}^{*}$.

Lemma 12 As before, let $\mathbf{x}=O P T(M, r)$ and $\mathbf{y}=\operatorname{Nash}(M, r)$. Then, $x_{i} \geq y_{i} / 2, \forall i$.

Proof: Let $p$ be the smallest index such that $\delta_{p}^{*}$ is 0 , and $m+1$ if no such index exists. Similarly, let $q$ be the smallest index such that $\delta_{q}=0$, and $m+1$ if no such index exists. Clearly, $p \geq q$. For all $i<q, \delta_{i}^{*}=\delta_{i} / 2$ and for $i \geq q, \delta_{i}^{*} \geq \delta_{i} / 2$. From the previous two lemmas, $x_{i}=\sum_{j=i}^{m} \delta_{j}^{*} \frac{1}{a_{i} \| \mathbf{v}_{\mathbf{j}} \mid}$ and $y_{i}=\sum_{j=i}^{m} \delta_{j} \frac{1}{a_{i}\left\|\mathbf{v}_{\mathbf{j}}\right\|}$. The lemma now follows. 
Lemma 13 If the latency functions are all linear, then there exists a 2SS strategy (s,s') such that

$$
C\left(\mathbf{s}, \mathbf{s}^{\prime}\right) \leq \max \left(\frac{4}{3+\alpha+\alpha^{3} / 32}, \frac{4}{3+\alpha+\alpha(1-\alpha) / 8}\right) C(\mathbf{x})
$$

Proof: The set $A$ is defined, as before, as the set of $i$ such that $x_{i} \geq y_{i}$. Let $x(A)=\beta r, y(A)=\beta^{\prime} r$. As before, the initial stackelberg assignment $\mathbf{s}$ is concentrated on $\bar{A}$, in such a way that $\mathbf{s}+\mathbf{t}=\mathbf{y}$, where $\mathbf{t}$ is the nash assignment induced by $\mathbf{s}$. The best 2 SS strategy would be to choose $\mathbf{s}$ so that after the first round, when $\mathbf{s}$ is transferred to elements of $A$, the remainder on $\bar{A}$ is assigned optimally. Because of the difficulty of analyzing this, we consider a different scheme below. Following earlier remarks, we will assume that $x(A)-y(A) \geq \alpha$.

The algorithm for 2SS depends on the following two cases, and entails specifying the assignments $\mathbf{s}, \mathbf{s}$. Choosing $s^{\prime}$ : In both the cases, the assignment $\mathbf{s}$ ' is constructed by performing the reassignment operation defined earlier, which involves adding a flow of $\alpha r$ to the nash assignment $\mathbf{y}_{A}$ on $A$.

We first derive a lower bound for $C\left(\mathbf{x}_{A}\right)$ before the choice of $\mathbf{s}$. As before, $\mathbf{z}_{A}$ is defined as $\mathbf{y}_{A}+\mathbf{s}_{A}$. Recall that $z_{i} \geq y_{i}, \forall i \in A$. Therefore, $C\left(\mathbf{z}_{A}\right) \geq\left(\beta^{\prime}+\alpha\right) r L(\mathbf{y})$. Let $\epsilon$ be such that $C\left(\mathbf{z}_{A}\right)=\left(\beta^{\prime}+\alpha+\epsilon\right) r L(\mathbf{y})$. By Lemma 2, $C\left(\mathbf{x}_{A}\right) \geq\left(\beta+\frac{\beta \epsilon}{\beta^{\prime}+\alpha}\right) r L(y)$.

We now consider different cases, and construct $\mathbf{s}$ separately in each of these.

Case 1: $\exists i \in \bar{A}$ such that $y_{i}-x_{i} \geq \alpha r$.

By Lemma 12, $y_{i} \leq 2 x_{i}$, which implies $x_{i} \geq y_{i}-x_{i} \geq \alpha r$.

First, suppose $b_{i} \geq \delta L(y)$. Define $\mathbf{z}_{\bar{A}}=\operatorname{Nash}\left(\bar{A}, r\left(1-\beta^{\prime}-\alpha\right)\right)$.

Choosing $s$ : choose $\mathbf{s}$ as $s_{j}=y_{j}-z_{j}, \forall j \in \bar{A}$ and $s_{j}=0, \forall j \in A$. By lemma $9, C\left(\mathbf{x}_{\bar{A}}\right) \geq(1-\beta-$ $\left.\frac{1-\beta^{\prime}-\alpha}{4}\right) r L\left(\mathbf{z}_{\bar{A}}\right)+b_{i} z_{i} / 4$. Since $y_{i}-x_{i} \geq \alpha r$, and $\mathbf{z}$ is the nash flow obtained by reducing a total of $\alpha r$ flow from $\bar{A}, z_{i} \geq x_{i} \geq \alpha r$. Therefore, $C\left(\mathbf{x}_{\bar{A}}\right) \geq \frac{3-4 \beta+\beta^{\prime}+\alpha+\delta \alpha}{4} L(\mathbf{z})$. Combining this with the lower bound for $C\left(\mathbf{x}_{A}\right)$, we have $C(\mathbf{x}) / C(\mathbf{z}) \geq \frac{3+\alpha}{4}+\frac{\delta \alpha+(1-\alpha) \epsilon}{4(1+\epsilon)}$. If $\epsilon \geq \alpha$, we get $C(\mathbf{x}) / C(\mathbf{z}) \geq \frac{3+\alpha+(1-\alpha) \alpha / 8}{4}$. If $\epsilon<\alpha$, we get $C(\mathbf{x}) / C(\mathbf{z}) \geq \frac{3+\alpha+\alpha^{3} / 32}{4}$, where we take $\delta=\alpha^{2} / 4$.

Next, suppose that $b_{i} \leq \delta L(\mathbf{y})$.

Choosing $s$ : In this case, choose $s_{i}=\alpha r$ and $s_{j}=0, j \neq i$. Define $\mathbf{z}_{\bar{A}}$ as $z_{i}=y_{i}-\alpha r$ and $z_{j}=$ $y_{j}, \forall j \in \bar{A} \backslash\{i\}$. Clearly, when $\mathbf{s}$ is removed from $\bar{A}$, the remainder on it is $\mathbf{z}_{\bar{A}}$. Let $x_{i}=\gamma r \geq \alpha r$ and $y_{i}=\gamma^{\prime} r \geq 2 \alpha r$. By lemma 9, $C\left(\mathbf{x}_{\bar{A} \backslash\{i\}}\right) \geq\left(1-\beta-\gamma-\frac{1-\beta^{\prime}-\gamma^{\prime}}{4}\right) r L(y)=\frac{3-4 \beta-4 \gamma+\beta^{\prime}+\gamma^{\prime}}{4} r L(y)$. Since $b_{i} \leq \delta L(y), \ell_{i}\left(z_{i}\right) \leq \frac{\gamma^{\prime}-\alpha+\alpha \delta}{\gamma^{\prime}} L(y)$ and $C\left(\mathbf{x}_{\{i\}}\right) \leq\left(\gamma^{\prime}-\alpha\right) \frac{\gamma^{\prime}-\alpha+\alpha \delta}{\gamma^{\prime}} L(y) . C\left(\mathbf{x}_{\{i\}}\right)=\gamma\left(a_{i} \gamma+b_{i}\right) \geq \frac{\gamma^{2}}{\gamma^{\prime}} L(y)$. Putting all of these together, we get

$$
C(\mathbf{x}) \geq \frac{3-4 \gamma+\beta^{\prime}+\gamma^{\prime}+4 \epsilon+4 \gamma^{2} / \gamma^{\prime}}{4} L(y) \quad \text { and } \quad C(\mathbf{z}) \leq\left(1+\epsilon-\alpha \frac{\gamma^{\prime}-\alpha}{\gamma^{\prime}}+\alpha \delta\right) L(y) .
$$

Thus, using $\gamma^{\prime} \geq 2 \alpha$, we get

$$
\frac{C(\mathbf{x})}{C(\mathbf{z})}-\frac{3+\alpha}{4} \geq \frac{\alpha^{2}-4 \gamma^{\prime} \alpha \delta+\gamma^{\prime}(1-\alpha) \epsilon}{4 \gamma^{\prime}(1+\epsilon)} .
$$

When $\epsilon>\alpha$, we get $\frac{C(\mathbf{x})}{C(\mathbf{z})} \geq \frac{3+\alpha+\alpha^{2}}{4}$, with $\delta$ set to $\alpha^{2} / 4$. When $\epsilon \leq \alpha$, we obtain

$$
\frac{C(\mathbf{x})}{C(\mathbf{z})}-\frac{3+\alpha}{4} \geq \frac{\alpha^{2}}{4(1+\alpha)}
$$


Case 2: $y_{i}-x_{i}<\alpha r, \forall i \in \bar{A}$.

In this case, there always exists a set $B \subset \bar{A}$ such that $\alpha r / 2 \leq y(B)-x(B) \leq \alpha r$. Let $x(B)=\gamma r, y(B)=$ $\gamma^{\prime} r$. Define $\mathbf{z}_{\bar{A} \backslash B}=N a s h\left(\bar{A} \backslash B, r\left(1-\beta^{\prime}-\gamma-\alpha\right)\right)$ and $\mathbf{z}_{B}=\mathbf{x}_{B}$.

Choosing $s$ : Choose s so that $s_{j}=y_{j}-x_{j}, \forall j \in B$ and $s_{j}=y_{j}-z_{j}, j \in \bar{A} \backslash B$. After the first round, when $\mathbf{s}$ is moved to $A$, assignment $\mathbf{z}$ remains on $\bar{A}$. By Lemma $9 C\left(\mathbf{x}_{\bar{A} \backslash B}\right) \geq\left(1-\beta-\gamma-\frac{1-\beta^{\prime}-\gamma-\alpha}{4}\right) L(\mathbf{z})$, which gives $C\left(\mathbf{x}_{\bar{A} \backslash B}\right) \geq \frac{3-4 \beta-3 \gamma+\alpha}{4} L(\mathbf{z}) . C\left(\mathbf{z}_{\bar{A} \backslash B}\right)=\left(1-\beta^{\prime}-\gamma-\alpha\right) L(\mathbf{z})$. Adding the above inequalities, we get

$$
C\left(\mathbf{x}_{M \backslash B}\right) \geq \frac{3-3 \gamma+\alpha+4 \beta \epsilon /\left(\alpha+\beta^{\prime}\right)}{4} L(\mathbf{z})
$$

Thus

$$
\frac{C\left(\mathbf{x}_{M \backslash B}\right)}{C\left(\mathbf{z}_{M \backslash B}\right)} \geq \frac{3-3 \gamma+\alpha+4 \beta \epsilon /\left(\alpha+\beta^{\prime}\right)}{4(1-\gamma+\epsilon)} \geq \frac{3+\alpha}{4}+\frac{\alpha \gamma+(1-\alpha) \epsilon}{4(1-\gamma+\epsilon)} .
$$

Since $C_{B}(\mathbf{x})=C_{B}(\mathbf{z}), \frac{C(\mathbf{x})}{C(\mathbf{z})}$ is bounded by the same ratio. By Observation $2, \gamma \geq \alpha / 2$. Thus, if $\epsilon \geq \gamma$, then

$$
\frac{C(\mathbf{x})}{C(\mathbf{z})} \geq \frac{3+\alpha+(1-\alpha) \alpha / 2}{4}
$$

else

$$
\frac{C(\mathbf{x})}{C(\mathbf{z})} \geq \frac{3+\alpha+\alpha^{2} / 2}{4}
$$

\section{Reverse Stackelberg Strategy}

In this section, we explore the model where the stackelberg assignment is made after the remaining $(1-\alpha) r$ fraction has formed a nash equilibrium. Clearly, the best stackelberg strategy would be to assign the $\alpha$ fraction optimally, given the remaining assignment. The lemma below shows that such a strategy is at least as good as 1SS. Let $\mathbf{t}$ be the nash assignment of the $1-\alpha$ fraction initially. Let $\mathbf{s}=O P T(M, \alpha r, \bar{\ell})$ be the subsequent stackelberg assignment, where $\bar{\ell}_{e}(u)$ is defined as $\ell_{e}\left(u+t_{e}\right), \forall e$. We still have the model of a network with two nodes and $m$ parallel links between them.

Lemma $14 C(\boldsymbol{s}+\boldsymbol{t}) \geq C_{1 S S}$, where $C_{1 S S}$ is the cost of the best strategy for 1SS.

Proof: Let $A=\left\{i: x_{i} \geq t_{i}\right\}$, where $\mathbf{x}$ is the optimal assignment, as before. Note that $x(\bar{A}) \leq t(\bar{A})=$ $r(1-\alpha)-t(A)$, which implies $r-x(\bar{A})-t(A)=x(A)-t(A) \geq \alpha r$. Consider an assignment $\mathbf{s}$, that assigns $\alpha r$ to elements in $A$, while keeping $s_{i}^{\prime}+t_{i} \leq x_{i}, \forall i \in A$, which can easily be done since $x(A)-t(A) \geq \alpha r$. Clearly, $C(\mathbf{x}) \geq C_{A}\left(\mathbf{s}^{\prime}+\mathbf{t}\right) \geq(t(A)+\alpha r) L$, where $L$ is the common nash latency of $\mathbf{t}$. If $C_{A}\left(\mathbf{s}^{\prime}+\mathbf{t}\right)=(t(A)+\alpha r+\epsilon r) L, C\left(\mathbf{s}^{\prime}+\mathbf{t}\right)=(1+\epsilon) r L$, and therefore, $C(\mathbf{x}) / C(\mathbf{z}) \geq \frac{t(A) / r+\alpha+\epsilon}{1+\epsilon} \geq \alpha$. 


\section{References}

[AP+01] D. Anson, D. Powell and M. Stein. A Game Theoretic Approach to Strategic Force Planning \& Strategic Stability Assessment. Technical Report, Los Alamos National Laboratory LA-UR 01-31-57 (2001).

[BMW56] M. Beckman, C. Mcguire and C. Winstein. Studies in the Economics of Transportation. Yale University Press, 1956.

[BPS99] J. Bennett, C. Partridge and N. Shectman. "Packet reordering is not Pathological Network Behavior," IEEE/ACM Transactions on Networking, 7(6), Dec. 1999, pp. 789-798.

[CR+93] J. Carrahan, P. Russo, K. Kitami and R. Kung. Intelligent Network Overview. IEEE Communications Magzine, 31, pp. 30-36, 1993.

[CS+93] R. Cocchi, S. Shenker, D. Estrin and L. Zhang. Pricing in Computer Networks: Motivation, Formulation and Example. IEEE/ACM Transactions on Networking. 1(6), pp. 614-627, 1993.

[CS00] T. O’Connell, and R. Stearns. Polynomial Time Mechanisms for Collective Decision Making. Game Theory and Decision Theory in Agent-Based Systems. S. Parsons, P. Gmytrasiewicz, P. and M. Wooldridge, (eds.), Kluwer Academic Publishers, 2000.

[DH95] S. Deering and R. Hinden. Internet Protocol Version 6 Specification. Internet Draft IETF March 1995.

[Du86] P. Dubey. Inefficiency of nash Equilibria. Mathematics of Operations, Research. 11(1), pp. 1-8, 1986.

[DS69] S. Dafermos and F. Sparrow. The Traffic Assignment Problem for a General Network. J. Research of the National Bureau of Standards Series B. 73B(2), pp. 91-118, 1969.

[ES91] A. Economides and J. Silvester. Multi-Objective Routing in Integrated Services Network: A Game Theory Approach. Proc. IEEE INFOCOM pp. 1220-1225, 1991.

[FPS00] J. Feigenbaum, C. Papadimitriou and S. Shenker. Sharing the Cost of Multicast Transmissions. Proc. 31 st Annual ACM Symposium on Theory of Computing (STOC). pp. 218-227, 2000.

[GJ79] M. Garey and D. Johnson. Computers and Intractability: A Guide to the Theory of NPCompleteness. Freeman, 1979.

[KLO97a] Y. Korillis, A. Lazar and A. Orda. Achieving Network Optima Using Stackelberg Routing Strategies. IEEE/ACM Transactions on Networking. 5(1), pp. 161-173, 1997.

[KP99] E. Koutsoupias and C. Papadimitriou. newblock Worst-Case Equilibria. Proc. 16th Annual Symposium on Theoretical Aspects of Computer Science (STACS), pp. 403-413, 1999.

[KLO97b] Y. Korillis, A. Lazar and A. Orda. Capacity Allocation Under Noncooperative Routing. IEEE Transactions on Automatic Control. 42(3), pp. 309-325, 1997.

[MS01] M. Mavronicolas and P. Spirakis. The Price of Selfish Routing. Proc. 33rd Annual ACM symposium on Theory of Computing. pp. 510-519, 2001.

[NR99] N. Nisan and A. Ronen. Algorithmic Mechanism Design. Proc. 31st Annual ACM Symposium on Theory of Computing (STOC). pp. 129-140, 1999.

[Ow95] G. Owen. Game Theory. Academic Press, 3rd Edition, 1995.

[ORS93] A. Orda, R. Rom and N. Shimkin. Competitive Routing in Multi-User Communication Networks. IEEE/ACM Transactions on Networking. 1, pp. 510-521, 1993.

[Pa01] C. Papadimitriou. Algorithms, games and the Internet. Proc. 33rd Annual ACM Symposium on Theory of Computing. pp. 749-753, 2001. 
[Ro01] T.Roughgarden. Stackelberg Scheduling Strategies. Proc. 31st ACM Symposium on Theory of Computing (STOC). pp. 2001.

[Ro01a] T.Roughgarden. Designing Networks for selfish users is hard. Proc. 31st ACM Symposium on Theory of Computing (STOC)??. pp. 2001.

[RT00] T.Roughgarden and E.Tardos. How bad is Selfish Routing. Proc. 41st Annual Symposium on Foundations of Computer Science. pp. 93-102, 2000.

[Ro02] T.Roughgarden. How Unfair is Optimal Routing. to appear in Proc. ACM-SIAM Symposium on Diceret Algorithms (SODA). 2002.

[SMG01] C. Saraydar, N. Mandayam and D. Goodman. Efficient Power Control via Pricing in Wireless Data Networks To Appear in IEEE Trans. on Communications, 2001.

[Se85] Y. Sheffi. Urban Transportation Networks: Equilibrium Analysis with Mathematical Programming methods. Prentice Hall, 1985.

[Sh95] S. Shenker. Making Greed Work in network: A Game-Theoretic Analysis of Switch Service Disciplines. IEEE/ACM Transactions on Networking. 3(6), pp. 819-831, 1995.

[Wa52] J. Wardrop. Some Theoretical Aspects of road Traffic Research. Proc. Institute of Civil Engineers, Part II, Vol. 1, pp. 325-378, 1952.

[Web] http://www.lanl.gov/orgs/d/d5/projects/MESA/mesa.html http://www.lanl.gov/orgs/d/d2/projects.html. 